mit

$$
\begin{aligned}
& a_{0}= \frac{\beta_{0}^{2}}{R^{2}}(C \lambda)_{00} \alpha_{00}\left(1-\frac{\beta_{0}^{2} D_{00}}{R^{2} \alpha_{00}}\right), \\
& a_{1}= {\left[\alpha _ { 0 0 } \frac { n \mu _ { 0 0 } } { ( n \mu ) _ { 0 0 } } \left\{\frac{\alpha^{\prime}}{\alpha_{00}}-\frac{\alpha D^{\prime}}{\alpha_{00} D}-\left(\frac{\mu^{\prime}}{\mu_{00}}-\frac{\mu \lambda^{\prime}}{\mu_{00} \lambda}\right)\right.\right.} \\
&\left.\left.\cdot\left(1-\frac{\beta_{0}^{2} D_{00}}{R^{2} \alpha_{00}}\right)-\frac{\beta_{0}^{2}(D+\chi)}{R^{2} \alpha_{00} T}\left(\frac{\lambda_{00}}{\lambda} \frac{\mu}{\mu_{00}}+1\right)\right\}\right]_{00}, \\
& a_{2}= {\left[\frac{D+\chi}{\lambda T_{-}} \frac{(n \mu)^{2}}{(n \mu)_{00}^{2}} \frac{\mu_{00}}{\mu}\left(\frac{D^{\prime}}{D}+\frac{1}{T_{-}}-\frac{(D+\chi)^{\prime}}{D+\chi}\right)\right]_{00} } \\
& \quad+\left[\frac{D+\chi}{\lambda T} \frac{n \mu}{(n \mu)_{00}}\right]_{00}\left[\left(\frac{\mu^{\prime}}{\mu}-\frac{\lambda^{\prime}}{\lambda}\right) \frac{n \mu}{(n \mu)_{00}}\right] .
\end{aligned}
$$

Hierin bedeuten die Abkürzungen für eine beliebige Ortsfunktion $F(r)$

$$
\begin{aligned}
F_{00} & =\frac{2}{R^{2}\left[J_{1}\left(\beta_{0}\right)\right]^{2}} \int\left[J_{0}\left(\beta_{0} \frac{r}{R}\right)\right]^{2} F^{(0)} r d r, \\
F^{\prime} & =\left.\frac{\partial F}{\partial T_{-}}\right|_{T^{(0)}}
\end{aligned}
$$

und ferner die Symbole: $j=$ elektrische Stromdichte der Elektronen; $E=$ elektrische Längsfeldstärke; $\mu=$ Elektronenbeweglichkeit; $\lambda=\mathrm{W}$ ärmeleitfähigkeit des Neutralgases; $D=$ effektiver Diffusionskoeffizient; $\chi=$ effektiver Thermodiffusionskoeffizient; $n=$ Elektronendichte; $C=$ Verhältnis von Elektronen- zu Neu- tralgastemperatur; $\beta_{0}=$ erste Nullstelle der $J_{0}$-BesseLFunktion. Der Index (0) kennzeichnet den stationären Zustand.

Ersichtlich wird dieses Kriterium durch Mittelwerte verschiedener Daten der stationären Lösung bestimmt.

Führt man die entsprechenden Größen der SснотткуLösung in das Kriterium ein, so ergibt eine Störungsrechnung für die $m=0$-mode Stabilität in Úbereinstimmung mit den experimentellen Erwartungen.

Die Auswertung des Kriteriums für höhere Ströme und Drucke erfordert die Kenntnis der entsprechenden stationären Lösung, der ,thermisch inhomogenen Plasmasäule“. Die charakteristischen Größen der thermisch inhomogenen Säule sind durch Lösung eines Eigenwertproblems für die beiden abhängigen Variablen der Elektronentemperatur und -dichte zu ermitteln. Wegen analytischer Schwierigkeiten wird das gekoppelte Differentialgleichungssystem nach geeigneter Umformung mit Hilfe eines Digitalrechners behandelt. Die Berechnungen sind noch nicht abgeschlossen. Sie lassen jedoch bereits erkennen, daß in dem Bereich der thermisch inhomogenen Plasmasäule Instabilität für die $m=0$-mode nach dem Kriterium (2) zu erwarten ist. Die Untersuchungen werden auch in der Richtung fortgesetzt, den notwendigen Charakter des angegebenen Kriteriums nachzuweisen.

\section{Zur Messung der Lumineszenzabklingzeiten von CsJ und CsJ/Tl nach Anregung mit Licht und $\alpha$-Teilchen}

\section{Von W. Rehmann, K. Reiber und A. Scharmann}

I. Physikalisches Institut der Universität Gießen

(Z. Naturforschg. 18 a, 1139-1141 [1963]; eingeg. am 7. September 1963)

In den vergangenen Jahren sind in einer Reihe von Arbeiten, die sich mit der Aufklärung des Lumineszenzmechanismus in reinem und Tl-aktiviertem CsJ befaßten, neben spektralen Untersuchungen Messungen über die Abhängigkeit der Lumineszenzabklingzeit von der Temperatur ${ }^{1-9}$, der Aktivatorkonzentration ${ }^{10}$ und der Anregungsdichte ${ }^{11-14}$ angestellt worden. Zur Erklärung des Temperaturgangs der Abklingzeit diente dabei ein Modell des Lumineszenzzentrums von BonaNoMi und Rosser ${ }^{1}$.

In Ergänzung zu diesen Untersuchungen sollen hier einige Ergebnisse aufgeführt werden, die bei der Mes-

1 J. Bonanomi u. J. Rossel, Helv. Phys. Acta 25, 725 [1952].

2 B. Hahn u. J. Rosser, Helv. Phys. Acta 26, 271, 803 [1953].

3 H. Knoepfel, E. Loepfe u. P. Stoll, Z. Naturforschg. 12 a, 348 [1957].

4 H. Enz u. J. Rossel, Helv. Phys. Acta 31, 25 [1958].

5 I. K. Pliavin', Opt. i Spektroskopiya 7, 41 [1959].

6 Z. L. Morgenshtern, Opt. i Spektroskopiya 7, 146 [1959].

7 I. K. Pliavin' u. M. F. TrinkLer, Opt. i Spektroskopiya 12, 367 [1962].

8 F. E. Senftele, P. Martinez u. V. P. Alekna, Rev. Sci. Instrum. 33, 819 [1962].

9 H. V. Watts, L. Reiffel u. M. D. Oestreich, Nuclear Electronics I, 3 [1962]. sung der Lumineszenzabklingzeit an CsJ-Kristallen mit unterschiedlichem Tl-Gehalt gewonnen wurden. Die Kristalle wurden dabei mit UV-Lichtblitzen und $\alpha$-Teilchen angeregt, um den Einfluß der Energieübertragung vom Anregungskanal zum Lumineszenzzentrum auf die Abklingzeit zu bestimmen.

\section{Experimentelles}

Die Messungen wurden an 5 CsJ-Kristallen mit unterschiedlichem Tl-Gehalt durchgeführt; die flammenphotometrische Bestimmung ergab folgende Tl-Gehalte: $0 \%, 0,03 \%, 0,15 \%, 0,37 \%$ und $0,4 \%$ (Angaben in Gew.Proz.). Von den Kristallen wurden Dünnschliffe von 0,1-0,2 mm Stärke nach einem von Knoepfel, Loepfe und STOLL ${ }^{15}$ beschriebenen Polierverfahren angefertigt.

Für die Experimente bei $\alpha$ - und UV-Anregung dienten zwei in ihrem Aufbau ähnliche Meßanordnungen. Dabei waren die Kristalle in einer evakuierbaren Kammer an einem Kupferblock befestigt, der mit flüssiger

10 Iu. A. Tsiruin, S. N. Komnick u. L. M. Solfer, Opt. i Spektroskopiya 6, 265 [1959].

11 R. S. Storey, W. JAck u. A. W don 72, 463 [1958].

12 A. Scharmann, Z. Phys. 157, 301 [1959].

13 M. Bormann, G. Andersson-Lindström, H. Neuert u. H. PolLEHN, Z. Naturforschg. 14 a, 681 [1959].

14 J. C. Robertson u. J. G. Lynch, Proc. Phys. Soc., London 77, 751 [1961].

15 H. Knoeprel, E. Loepfe u. P. Stoll, Helv. Phys. Acta 30, 521 [1957]. 
Luft auf tiefe Temperaturen gebracht oder mittels einer eingelassenen Heizwendel aufgeheizt werden konnte. Seine jeweilige Temperatur wurde mit einem KupferKonstantan-Thermoelement bestimmt.

Für die Anregung mit $\alpha$-Teilchen wurde ein $1 \mu \mathrm{c}$ Po 210-Präparat benutzt. Die Aufnahme der Lumineszenzblitze erfolgte durch ein Quarzfenster der Vakuumkammer mit einem PSEV vom Typ R C A 6810 A; zwischen PSEV und Quarzfenster konnten zum Ausfiltern verschiedener Lumineszenzbanden Absorptionsfilter angebracht werden. Der Arbeitswiderstand des PSEV war so gewählt, daß die $R C$-Zeit des Übertragungsnetzwerks klein gegen die zu messende Abklingzeit war. Die erhaltenen Spannungsimpulse wurden mit einem Tektronix-Oszillographen vom Typ $545 \mathrm{~A}$ aufgenommen, photographiert und ausgewertet.

Bei den Messungen mit UV-Anregung erhielt die Vakuumkammer ein zweites Quarzfenster, durch das das Anregungslicht eingestrahlt wurde. Die UV-Blitze wurden mit einer selbstgebauten Blitzlampe von $2 \cdot 10^{-8}$ sec Blitzdauer erzeugt ${ }^{16}$. Das Spektrum der UV-Blitze war durch ein Absorptionsfilter auf den Bereich zwischen $2700 \AA$ und $3700 \AA$ begrenzt.

\section{Ergebnisse}

Aus wäßriger Lösung gezogene CsJ-Kristalle zeigen nach Untersuchungen von MoRgenshtern ${ }^{17}$ nach Anregung mit $\alpha$-, $\beta$ - und $\gamma$-Strahlen bei tiefen Temperaturen eine Emissionsbande zwischen $3000 \AA$ und $3900 \AA$ A die sogenannte „kalte“ Komponente. Mit Licht einer Wellenlänge $>2000 \AA$ kann diese „kalte“ Komponente nicht angeregt werden. (Bei Kristallen, die wie in unserem Fall aus der Schmelze gezogen wurden, tritt daneben bei $\alpha$-, $\beta$-, $\gamma$ - und Lichtanregung $(2400 \AA)$ eine Emission im Bereich von $3600 \AA$ bis $5000 \AA$ auf. Die Intensität dieser sogenannten „blauen“ Komponente ist im Temperaturbereich von $-190^{\circ} \mathrm{C}$ bis $+20^{\circ} \mathrm{C}$ angenähert konstant und ist um so größer, je geringer die „kalte“ Komponente in einem Kristall vertreten ist.)

Aktivierung der Kristalle mit $\mathrm{Tl}$ bringt bei höheren Konzentrationen die „kalte“ Komponente zum Verschwinden. Dafür tritt nunmehr im gesamten untersuchten Temperaturbereich eine Bande zwischen $5000 \AA$ und $7000 \AA$ auf, die „warme“ Komponente. Das Optimum ihrer Intensität liegt ungefähr bei Zimmertemperatur.

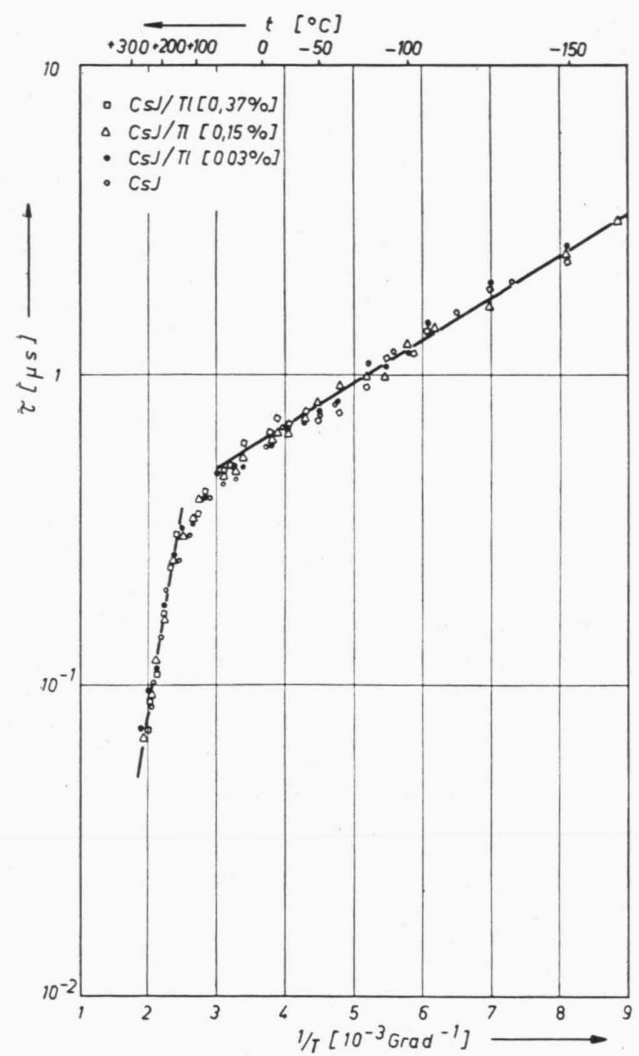

Abb. 1. Abklingzeit der „warmen“ Komponente von CsJ mit unterschiedlichem Tl-Gehalt als Funktion der reziproken Temperatur. Anregung: UV-Blitz.

${ }^{16}$ K. ReIBER, Diplomarbeit, Gießen 1963.

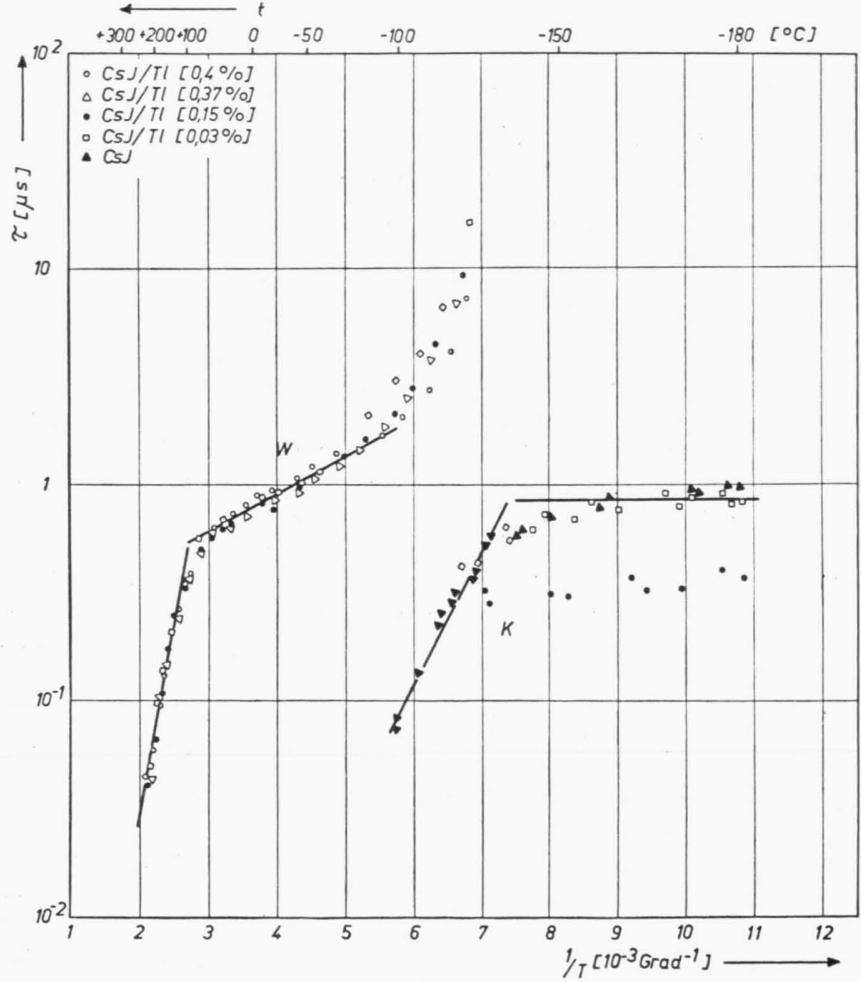

Abb. 2. Abklingzeit von CsJ mit unterschiedlichem Tl-Gehalt als Funktion der reziproken Temperatur. Anregung: $\alpha$-Teilchen (Po 210). W: „Warme“ Komponente, K: „Kalte“ Komponente.

17 Z. L. Morgenshtern, Opt. i Spektroskopiya 8, 355 [1960]. 
In allen Fällen klingt die Lumineszenz der CsJ- und $\mathrm{CsJ} / \mathrm{Tl}-\mathrm{Kristalle}$ exponentiell ab: $\quad I=I_{0} \exp \{-t / \tau\}$; $\tau$ ist die Lumineszenzabklingzeit.

In Abb. 1 und Abb. 2 ist $\tau$ logarithmisch als Funktion der reziproken absoluten Temperatur dargestellt. Abb. 1 zeigt, daß die Änderung des Tl-Gehaltes das Abklingen der „warmen“ Komponente bei UV-Anregung nicht beeinflußt. Die gemessene Kurve kann im gesamten Temperaturbereich durch zwei Geraden angenähert werden. Dagegen weicht die bei $\alpha$-Anregung aufgenommene Kurve dieser „warmen“ Komponente bei tieferen Temperaturen von diesem Verlauf ab: Die Abklingzeiten sind hier länger als bei UV-Anregung. Außerdem zeigt sich in diesem Bereich ein Einfluß der Aktivatorkonzentration auf den Wert von $\tau$, und zwar nimmt dieser mit wachsendem Tl-Gehalt der Kristalle ab.

Es sei noch darauf hingewiesen, daß bei UV-Anregung die „warme“ Komponente auch an dem nicht aktivierten Kristall zu beobachten war. Bei $\alpha$-Anregung tritt diese Bande ebenfalls an dem CsJ-Kristall auf, entzieht sich jedoch wegen ihrer geringen Lumineszenzintensität einer Messung.

\section{Diskussion}

Der exponentielle Verlauf der Abklingkurven deutet auf einen Leuchtprozeß mit einer Reaktionskinetik 1. Ordnung hin. Für einen solchen Vorgang entwickelten Bonanomi und Rossel ${ }^{1}$ ein Modell des Lumineszenzzentrums, das die Temperaturabhängigkeit der Abklingzeit erklärt. Dabei wird angenommen, daß sich im angeregten Lumineszenzzentrum ein Elektron in einem metastabilen Zustand befindet; von hier wird es entweder durch die - thermisch zugeführte - Aktivierungsenergie $E_{1}$ in einen lumineszenzfähigen Zustand gebracht und geht anschließend unter Emission in den Grundzustand über, oder es kann unter Aufnahme der Aktivierungsenergie $E_{2}$ strahlungslos den Grundzustand erreichen. Unter diesen Voraussetzungen ergibt sich für die Abklingzeit folgende Gesetzmäßigkeit:

$$
\tau=\frac{1}{s_{1} \exp \left\{-E_{1} / k T\right\}+s_{2} \exp \left\{-E_{2} / k T\right\}}
$$

Die logarithmische Darstellung der Abklingzeit über der reziproken Temperatur muß demnach eine Kurve ergeben, die durch zwei Geraden verschiedener Neigung näherungsweise wiedergegeben werden kann. Aus der Lage der Geraden kann man die Größen $E_{1,2}$ und $s_{1,2}$ entnehmen. Die bei UV-Anregung gemessene Kurve (Abb. 1) entspricht dem hier geforderten Verlauf; die Abweichung der bei $\alpha$-Anregung aufgenommenen Werte (Abb. 2) dürfte auf eine Verzögerung während des Energietransportes vom Anregungskanal zum Lumineszenzzentrum zurückzuführen sein; diese Annahme wird gestützt durch die Beobachtung, daß in dem gleichen Temperaturbereich die Abklingzeit bei höherer Aktivatorkonzentration, d. $\mathrm{h}$. bei kleinerem mittlerem $\mathrm{Ab}$ stand vom Anregungskanal zum Zentrum, kürzer wird.

Aus den gemessenen Kurven ergeben sich für die "warme" Komponente folgende Werte:

$$
\begin{aligned}
& \text { bei UV-Anregung } E_{1}=0,027 \mathrm{eV}, s_{1}=5 \cdot 10^{6} \mathrm{~s}^{-1} \text {, } \\
& E_{2}=0,26 \mathrm{eV}, \quad s_{2}=10^{10} \mathrm{~s}^{-1} \text {; } \\
& \text { bei } \alpha \text {-Anregung } \quad E_{1}=0,034 \mathrm{eV}, \quad s_{1}=5 \cdot 10^{6} \mathrm{~s}^{-1} \text {, } \\
& E_{2}=0,36 \mathrm{eV}, \quad s_{2}=10^{11} \mathrm{~s}^{-1} \text {. }
\end{aligned}
$$

(Setzt man nach Enz und Rosser ${ }^{4}$ für die wahrscheinlich auf einem Excitonenzerfall beruhende "kalte“ Komponente einen ähnlichen Leuchtprozeß an, so erhält man hier: $E_{1}{ }^{\prime}=0, \quad s_{1}{ }^{\prime}=1,2 \cdot 10^{6} \mathrm{~s}^{-1}, \quad E_{2}{ }^{\prime}=0,13 \mathrm{eV}$, $s_{2}^{\prime}=5 \cdot 10^{10} \mathrm{~s}^{-1}$.)

Das Auftreten der „warmen“ Komponente auch an nicht dotierten Kristallen darf als eine Bestätigung der Annahme von Knoepfel, Loepfe und Stoll ${ }^{15}$ angesehen werden, daß die langwellige Lumineszenzbande im $\mathrm{CsJ} / \mathrm{Tl}$ auf J-Lücken im Kristallgitter zurückzuführen ist, die durch Tl-Einbau, jedoch auch durch eine thermische Vorbehandlung der Kristalle, geschaffen werden können. Bei unseren Kristallen kann damit das Auftreten der „warmen" Komponente am CsJ auf die thermische Vorgeschichte der aus der Schmelze gezogenen Kristalle zurückgeführt werden.

Herrn Professor Dr. W. HanLe danken wir für stete Förderung, Herrn Dipl.-Phys. H. Hartmann für Unterstützung bei den Messungen. Weiterhin danken wir Herrn Dr. Nitschmann von der Firma E. L e i t z (Wetzlar) und dem Bundesministerium für wissenschaftliche Forschung für Zuwendungen. 